$62^{\text {ème }}$ Congrès de la SFCO, 02020 (2014)

DOI: $10.1051 /$ sfco/20146202020

(C) Owned by the authors, published by EDP Sciences, 2014

\title{
Tumeur adénomatoïde odontogène associée à un kyste dentigère à localisation intrasinusienne : à propos d'un cas.
}

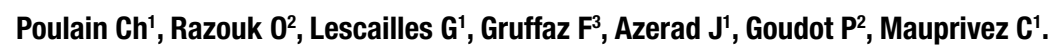

1. Service d'Odontologie, Groupe Hospitalier Pitié-Salpétrière, 47-83 Bd de l'Hôpital, 75013 Paris, FRANCE

2. Service de Stomatologie et de Chirurgie Maxillofaciale, Groupe Hospitalier Pitié-Salpétrière, 47- 83 Bd de l'Hôpital, 75013 Paris, FRANCE

3. Laboratoire d'Anatomo-pathologie et de Cytologie, Groupe Hospitalier Pitié-Salpétrière, 47-83 Bd de l'Hôpital, 75013 Paris, FRANCE

La tumeur odontogène adénomatoïde (TOA) est une tumeur bénigne rare qui représente 2,2 à 7,1\% de l'ensemble des tumeurs odontogènes. Décrite pour la première fois en 1905 par Steenlands [HARNET, 2012], elle a été pendant de nombreuses années considérée comme une variante de l'améloblastome. Aussi, elle a été initialement appelée adéno-améloblastome, du fait qu'à l'examen histologique, la lésion est formée par des cellules cylindriques rappelant les cellules améloblastiques formant des structures pseudoglandulaires remplie d'un matériel amorphe. Ce n'est qu'en 1969, Philipsen et Birn, ont introduit le terme de TOA ; cette appellation est adopté en 1971 par OMS et depuis utilisé dans la classification des tumeurs odontogènes [OMS, 2005]. Trois variantes clinico-topographiques sont identifiés : folliculaire, extrafolliculaire et périphérique [DE MATOS, 2012]. La forme folliculaire est la plus fréquente ( $71 \%$ des cas), il s'agit d'une lésion intra-osseuse associée à une dent incluse. Le type extrafolliculaire est de siège intraosseux et en rapport avec l'apex ou la racine d'une dent sur l'arcade. Le type périphérique très rare ( $3 \%$ des cas) est extraosseux et localisé au niveau de la gencive. Nous rapportons le cas d'une TOA associée à un volumineux kyste dentigère localisé dans le sinus maxillaire.

II s'agit d'une femme de 57 ans, édentée totale, qui s'est présentée pour une douleur sous-prothétique au niveau du maxillaire dans le secteur postérieur droit. L'examen clinique révèle une tuméfaction inflammatoire d'environ $2 \mathrm{~cm}$ dans le fond du vestibule associée à une ulcération traumatique. Le reste de l'examen est non contributif. Les examens radiographiques (orthopantomogramme et tomodensitométrie du massif facial) révèlent la présence d'une lésion ostéolytique uniloculaire comblant la totalité de la cavité sinusienne droite, à contours nets, et associée à une dent incluse. La lésion englobe la totalité de la couronne et les racines. A proximité de la dent incluse, de discrets microfoyers radioopaques sont objectivés. Divers diagnostics sont évoqués : une tumeur odontogène adénomatoïde, une tumeur épithéliale calcifiante ou tumeur de Pindborg, et un fibrome améloblastique (en raison de la dent incluse associée à des calcifications). L'énucléation de la lésion est pratiquée sous anesthésie générale. L'examen anatomopathologique identifie une TOA circonscrite dans la paroi d'un kyste dentigère. Une surveillance clinique et radiologique est mise en place.

Malgré la rareté de cette association (seize cas publiés dans la littérature), ce cas illustre l'importance d'une exérèse totale pour l'établissement du diagnostic de certitude. Le clinicien doit toujours garder à l'esprit de la possibilité de plusieurs entités pathologiques au sein d'une même lésion [ZHANG, 2010] et/ou du risque potentiel de transformation histologique voire de transformation maligne [PIRKLBAUER, 2012].

POULAIN Christophe

poulainchristophe.29@orange.fr

This is an Open Access article distributed under the terms of the Creative Commons Attribution License 4.0, which permits unrestricted use, distribution, and reproduction in any medium, provided the original work is properly cited. 